Revue critique de fixxion française contemporaine

22 | 2021

Figures du mensonge et de la mauvaise foi dans le roman contemporain

\title{
L'impossible sincérité du curateur. Authenticité, crédit et récit de soi dans les versions contemporaines du récit de collection
}

Loïse Lelevé

\section{CpenEdition}

Journals

Édition électronique

URL : https://journals.openedition.org/fixxion/323

DOI : $10.4000 /$ fixxion.323

ISSN : 2295-9106

Éditeur

Ghent University

Référence électronique

Loïse Lelevé, «L'impossible sincérité du curateur. Authenticité, crédit et récit de soi dans les versions contemporaines du récit de collection », Revue critique de fixxion française contemporaine [En ligne], 22 | 2021, mis en ligne le 15 juin 2021, consulté le 17 février 2022. URL : http://journals.openedition.org/ fixxion/323; DOl : https://doi.org/10.4000/fixxion.323

Ce document a été généré automatiquement le 17 février 2022.

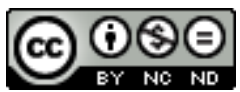

Les contenus de la Revue critique de fixxion française contemporaine sont mis à disposition selon les termes de la licence Creative Commons Attribution - Pas d'Utilisation Commerciale - Pas de Modification 4.0 International. 


\title{
L'impossible sincérité du curateur. Authenticité, crédit et récit de soi dans les versions contemporaines du récit de collection
}

\author{
Loïse Lelevé
}

Ce qu'on pourrait appeler le récit de collection connaît donc son plein développement entre les années 1840 et le début du XXe siècle, tombe ensuite en désuétude, mais semble faire une réapparition dans la littérature contemporaine européenne, au prix c'est ce que nous entendons montrer dans le présent article - de plusieurs déplacements, à commencer par la relégation de son protagoniste au rang de curateur ${ }^{1}$ chargé d'authentifier une collection. C'est une figure inédite par rapport aux récits du $\mathrm{XIX}^{\mathrm{e}}$ siècle : il n'est en effet ni collectionneur à proprement parler (il ne possède pas la collection qui lui est confiée), ni historien de l'art, personnage qui fait l'objet d'un traitement sui generis dans la littérature contemporaine ${ }^{2}$. Il se situe plutôt entre le connaisseur, l'expert et l'esthète : dépositaire d'un savoir autodidacte ou dont on ne connaît pas l'origine, il n'est pas recruté par des institutions mais par des collectionneurs privés peu recommandables, pour en authentifier les œuvres. Pourtant, comme les collectionneurs des années 1840 , il se caractérise par son caractère asocial et son obsession pathologique pour la collection dont il a la garde. Néanmoins celle-ci est désormais minée de part en part par la menace du faux, des objets inauthentiques qui la constituent à la fausseté du discours de savoir qu'elle prétend tenir sur ses propres pièces comme sur le monde. Les faux de la collection ne sont pas dans les récits contemporains le résultat d'erreurs (d'attribution, de catalogage) ni même d'une manipulation délibérée, mais le symptôme d'un dysfonctionnement inhérent à la collection elle-même. Là où, dans la culture humaniste, le cabinet d'amateur ou la Wunderkammer agissaient comme un microcosme reflétant et résumant le monde et l'étendue des connaissances disponibles à son sujet, là où la collection du XIXe siècle se présentait comme un résumé de l'histoire de l'art occidental (sur le modèle de celle de Nemo) ou comme un exposé des connaissances et des trouvailles d'un spécialiste (sur le 
modèle de la collection de faïences d'un Champfleury, expert reconnu dans son domaine), la collection dans les romans contemporains ne fonctionne plus que comme un ensemble de signes qui tournent à vide. C'est un trompe-l'œil : un dispositif qui imite des systèmes de construction du savoir et d'attestation de l'authenticité pour mieux en révéler la fragilité, l'inadéquation, le caractère falsifiable. Dès lors, le curateur ne peut plus être qu'un imposteur obligé ${ }^{3}$ : lui qui doit attribuer des noms et garantir des origines, le voilà prisonnier d'une somme de récits contradictoires, puisque ni les noms ni les histoires de provenance des faux n'ont plus de référent stable hors de la fiction que construit la collection. L'écriture, par les narrateurs-curateurs, de la collection (sous la forme de l'inventaire qui identifie et authentifie les objets) devient écriture du doute et, dès lors, écriture de soi : leurs notices de catalogue, détournées de leur but initial, se font lettres ou journaux intimes, la crise de l'authenticité de la collection provoquant en retour la crise de l'identité des curateurs. Dès lors que, dans la collection, l'étiquette ne peut plus correspondre à l'objet, toute conception de la vérité sous la forme traditionnelle de l'adaequatio rei et intellectus devient obsolète. Pour échapper à l'angoisse d'un monde de purs simulacres coupés de tout référent réel, il faut exhiber ou remotiver les signes devenus vides, quitte à leur imposer un référent nouveau, à le construire ad hoc. Le danger du mensonge n'est plus tant celui de la fausse identification ou de la fausse identité, dans le but de tromper, que celui d'une parole "cloisonnée" ${ }^{4}$, comme l'écrit le protagoniste du roman De toutes pièces de C. Portier, d'un rapport artificiellement figé entre les mots et les choses, qui empêche la resémantisation des signes et la réinvention de soi. Aussi, face au manque de sens de la collection, au délitement de leur identité, les curateurs narrateurs se construisent-ils des masques en série, selon des règles précises, et tentent-ils ainsi de "se montrer radic[aux] dans la maitrise de l'imposture" ${ }^{5}$, de convertir leur mauvaise foi en une hypocrisie (au sens étymologique, jouer à être un autre) avouée, mise en scène, performée $^{6}$. Se joue ainsi l'un des horizons éthiques possibles que dessine le roman contemporain dans son rapport à la vérité, à travers la tentative de construire de fragiles relations de confiance entre narrateurs et narrataires - autant de pactes de lecture, de pactes de fictionnalité en abyme, obliques, fuyants, mais proposant une forme de crédit narratif. Pour en étudier les mécanismes, nous nous appuierons principalement sur deux romans, De toutes pièces, de Cécile Portier (2018) et Athena, de John Banville (1995), sans nous interdire un détour par les récits de Georges Perec ou de Pierre Senges.

\section{Le récit de collection contemporain. Disparition de la collection, avènement du curateur aliéné}

2 Dès le XIX ${ }^{e}$ siècle, les œuvres mettent en scène la disparition de la collection quand celle-ci est conçue comme un espace de projection du désir et le portrait indirect d'un collectionneur maniaque mais sincère. Ce topos du roman de collection est repris de manière ironique et ludique à l'ère postmoderne dans la mise en scène d'enfouissements ou de dilapidations de collections désormais marquées par une inauthenticité foncière. Ainsi, dans Un cabinet d'amateur de Perec, le tableau éponyme, résumé en abyme de la collection d'Hermann Raffke, est enterré avec son possesseur, dans une reprise ironique de Vingt mille lieues sous les mers. La citation de la description de la collection de Nemo qui sert d'exergue au roman de Perec $^{7}$ vaut autant comme 
avertissement au lecteur que comme hommage à Verne : l'idéal de la collection-musée, synthèse à elle seule de l'ensemble de l'histoire de l'art occidental, est dépassé. Tous les tableaux ou presque de la collection Raffke sont faux, et le Cabinet d'amateur qui les représente est un instrument de vengeance. Dans le texte de Perec, l'onomastique fonctionne comme un piège linguistique: non seulement les noms de peintres et d'œuvres sont privés de tout fonctionnement référentiel correct ${ }^{8}$ et ne renvoient plus à des œuvres réelles mais à des tableaux fictifs, créés selon des règles de génération avant tout textuelles ${ }^{9}$; mais ils sont aussi le support d'un système intertextuel d'“implicitations" où la référence explicite en dissimule toute une série d'autres ${ }^{10}$, à commencer par les allusions "encryptées" à La vie mode d'emploi qui lui sert d'hypotexte. Avec la perturbation du système de la référence et le fonctionnement autotélique du texte, c'est toute l'articulation de la collection au monde, sa conception comme dispositif de représentation et de lecture d'une histoire de l'art cohérente, qui est remise en cause. Le cabinet d'amateur ne peut plus être le microcosme synthétisant, dans un idéal humaniste, les curiosités remarquables, culturelles et naturelles, de notre monde: il devient le genre esthétique, emprunté, par un art de l'ekphrasis ludique, au domaine pictural, d'exploration des possibles de la création littéraire, à commencer par le "fairesemblant"11. Au mensonge au service de la mystification mise en place, dans la diégèse, par Raffke, correspond donc, sur le plan métatextuel, une pratique littéraire du trompe-l'œil ${ }^{12}$, dans lequel le plaisir de l'illusion comme imitation (de discours savants sur l'histoire de l'art), masque autant qu'il l'exhibe l'angoisse du manque, du tarissement, de la répétition insensée, et se dévoile comme une tentative de "retrouver l'invention au-delà de l'énumération, le jaillissement au-delà de la citation, la liberté au-delà de la mémoire" ( $C A$ 28). La fiction perecquienne se présente dès lors moins comme une pratique du "mentir-vrai" où l'activité de fabulation trouverait sa justification éthique ou épistémique dans la modélisation herméneutique du monde qu'elle rendrait possible - et sans qu'il y ait donc, à proprement parler, de mensonge, d'intention de tromper, de la fiction -, que comme un jeu de simulation où l'angoisse de la coupure référentielle avec le réel, où l'anxiété liée à la dislocation des savoirs, est conjurée par l'exhibition, ironique et ludique, du simulacre comme tel. Le simulacre ne s'y substitue donc pas au réel dont il serait devenu indiscernable, comme chez Baudrillard ; mais, comme dans le trompe-l'œil, le vertige de la confusion possible entre faux et réalité se renverse toujours en plaisir de l'exhibition, a posteriori, du mécanisme de la tromperie.

3 À l'époque contemporaine, une mélancolie ${ }^{13}$ mortifère vient ainsi doubler le processus ludique de création d'une collection fictive. Dans Veuves au maquillage (2000) de Pierre Senges, le protagoniste, cherchant à se suicider, décide de confier la découpe méthodique de son corps en "lotissements" à des veuves homicides. Se constitue ainsi ironiquement une collection dont la finalité même est la disparition : les fragments de corps sont soigneusement prélevés selon un protocole qui emprunte autant aux traités d'Ambroise Paré qu'à l'Anatomie de la mélancolie de Burton. Leur devenir fait l'objet d'hypothèses qui fonctionnent comme autant de matrices narratives: les veuves et le narrateur s'amusent à en faire des "colis" piégés à envoyer par vengeance à des victimes désignées :

Nos petits colis sont prétextes à racontars, à billevesées : entre les veuves et moi [...] s'est établi un jeu : c'est à qui rapportera l'histoire la plus longue, la plus complète, la plus prometteuse - le compte-rendu qui satisfait le plus notre appétit de vengeance et de merveille. C'est à qui prétendra (à tort ou à raison : mais nous 
accordons toujours notre crédit aux témoins) avoir adressé le colis le plus fantasque au ministre le plus en vue [...] ; c'est à qui proposera le projet invraisemblable mais efficace, s'inventera une victime qui nous agrée ou nous dépasse, et la désigne parmi la foule. ${ }^{14}$

4 Ainsi les opérations de constitution de la collection sont-elles ironiquement réparties entre le narrateur et les veuves : lui fournit à la fois la matière première, et l'expertise de ses lots ; elles prélèvent, acquièrent, et cèdent ses membres. Le narrateur devient de la sorte une figure absurde de curateur volontairement aliéné à des collectionneuses qui ne possèdent rien. Dans ces conditions, la référence à Burton est significative d'un nouvel investissement du rapport entre le corps (figure microcosmique) et un macrocosme réinventé. La vivisection comme leçon d'anatomie est aussi une opération de connaissance de soi et du monde, à ceci près qu'il ne s'agit plus de faire découvrir le monde tel qu'il existerait en dehors de la fiction, mais de mettre en œuvre la constitution de mondes fictionnels et l'émergence ironique d'une figure d'auteur par suppression de soi-même du narrateur et récit de cette suppression ${ }^{15}$. La collection conserve une fonction de représentation herméneutique du monde (chaque fragment de corps est prétexte à histoires, à rappels théoriques et à investigations), cependant celle-ci est de part en part médiée par des références littéraires : c'est une anatomie de la culture et de la fiction ${ }^{16}$ qui s'y joue. Veuves au maquillage est dès lors exemplaire du récit de collection à la première personne, marqué par l'avènement d'un narrateur hypocrite, ironique et désabusé :

seul nous convient un raisonnement rétrospectif et volontairement de mauvaise foi : à l'origine de notre vie commune il y a (voici ce que je dicte aux veuves, elles se montrent à ce sujet aussi hypocrites que moi) cette décision, prise par moi seul, en mon pouvoir égotique et régalien, de procéder par amour, par noblesse et pour la farce, à l'effeuillage d'une marguerite à quarante-neuf pétales, celle de mon propre corps - un peu, beaucoup, passionnément. ${ }^{17}$

Seul le mensonge fictionnel, un mensonge qu'on ne saurait rédimer par l'excuse d'un "mentir-vrai ${ }^{18 "}$, permet ainsi de conserver sa valeur épistémique à la collection et à sa mise en récit. La création fictionnelle n'est en effet pas représentée dans le roman comme une simple entreprise de fabulation heuristique, mais comme une mise en règle du mensonge : la "mauvaise foi", devenue non seulement volontaire, mais codifiée sous la forme d'un jeu (presque) sérieux du narrateur et de ses complices, s'y transforme en hypocrisie ${ }^{19}$, en fabrication d'identités en série. Les disjecta membra du narrateur sont l'occasion de l'invention d'autant de masques, dans ce récit de soi sous forme de récit d'un suicide raffiné. Il se produit alors un renversement de la conception augustinienne du mensonge: "Augustin caractérise le mensonge par la nature de l'intention et sa finalité : on peut vouloir dire le faux, pour ne pas tromper autrui, ou dire le vrai pour l'induire en erreur, et cela, soit pour son bien, soit pour lui nuire. [...] Il s'agit donc, pour ne pas mentir, de dire ce que nous croyons être vrai, avec l'intention de faire croire ce que nous disons" ${ }^{20}$. À l'inverse, il s'agit, pour les narrateurs des récits de collection contemporains, de dire ce qu'ils savent ne pas être vrai, dans l'intention non de faire croire, mais d'inciter à accorder crédit, "un peu, beaucoup, passionnément", à ce qu'ils disent. Là où le mensonge "ne pouvait être qu'une péripétie, attisant la promesse d'une parousie renouvelée de la vérité" 21 , il devient l'enjeu même de ce qui se dessine comme une paradoxale éthique, au croisement de l'amour, même mortifère, de la noblesse (de l'imposture radicale) et de la farce. 
$6 \quad$ Et tandis que le narrateur de Senges se fait sciemment disparaitre pour reconstruire ironiquement une figure d'auteur, d'autres curateurs aliénés font de leur rapport à la collection l'enjeu de la difficile construction, sous l'égide de cette hypocrisie, de leur identité. C'est le cas, notamment, dans les romans de Banville et Portier.

\section{La collection, portrait inauthentique de menteurs mélancoliques}

7 Les récits de collection contemporains sont donc marqués par la permanence des systèmes de représentation humanistes du monde (cabinets d'amateur, anatomies). Mais l'idée que le microcosme de la collection demeure un reflet possible d'un macrocosme extérieur à la fiction qu'elle construit n'est maintenue que par goût de la supercherie. La collection devient moins un dispositif de savoir que le spectacle d'un chaos mélancolique et le portrait de personnages instables à travers des miroirs déformants. C'est qu'elle ne dépend plus d'un lien intime avec un collectionneur ${ }^{22}$, mais du regard que pose sur elle un curateur à la recherche de soi. La lettre et le journal, écritures de l'intime, sont alors, comme dans Veuves au maquillage, des formes privilégiées de ces récits.

8 Athena est le troisième tome de la "trilogie artistique" de Banville. Dans le premier, le narrateur, Freddie Montgomery, tombe amoureux d'un portrait féminin du siècle d'or hollandais, entreprend de le voler, et massacre à coups de marteau la jeune femme qui le surprend en flagrant délit. Le roman se présente comme une lettre adressée à son juge pour tenter de rendre compte de son geste, que le narrateur attribue à un défaut d'imagination: "This is the worst, the essential sin, I think [...]: that I never imagined her vividly enough, that I did not make her live. Yes, that failure of imagination is my real crime [...]. I could kill her because for me she was not alive" ${ }^{23}$. Dans le second tome, après avoir passé dix ans en prison, Montgomery devient expert autodidacte de peinture hollandaise et se donne la tâche de se racheter en réussissant là où il avait échoué : enfanter, de son imagination, une femme qui existe hors de son solipsisme effréné. Le roman se conclut sur une forme d'échec ("I am, I realise, only at the beginning of this birthing business" ${ }^{24}$ ), et Athena le voit plus tard, sous le pseudonyme de Morrow, chargé d'authentifier une collection de huit peintures flamandes, tout en étant amoureux de la mystérieuse "A." (comme art et comme Athéna). Seulement, sept des huit tableaux se révèlent faux, et $A$. n'est autre que la sœur du commanditaire de l'expertise, qui séduit Morrow pour mieux l'égarer. Ironiquement, le seul tableau authentique s'intitule The Birth of Athena. L'incertitude des lecteurs quant à la réalité de l'existence d'A. (est-elle le fruit de l'imagination de Freddie ou une femme réelle, manipulatrice ${ }^{25}$ ?) résume toute l'ambiguïté de cette conclusion de la trilogie : faisant l'expérience de la perte consentie (il laisse A. s'enfuir) comme de la désillusion artistique (puisque les œuvres sont fausses), Freddie, nouveau Zeus tirant Athéna de son crâne, parvient-il à donner vie, par sa lettre, à un autre féminin, et donc à achever sa rédemption ${ }^{26}$ ? Ou, prisonnier de ses illusions, ne fait-il que répéter les erreurs (fascination pour l'art comme seul monde possible d'existence, réification des femmes par un désir voyeuriste, mauvaise foi d'un être se scrutant sans cesse) des premiers tomes $^{27}$ ?

9 Freddie est en tout cas un exemple parfait de narrateur non fiable : il masque son identité (seul le nom Morrow apparaît dans Athena, c'est au lecteur de reconnaître le 
personnage sous le pseudonyme, à travers des indices dissimulés avec plus ou moins d'hypocrisie ${ }^{28}$ ), et ment sans cesse, sans - paradoxalement - jamais s'en cacher ${ }^{29}$. Il ne cesse de commenter les travers de son discours, disséquant tout ce que celui-ci peut avoir d'artificiel, déréalisant les événements, les souvenirs et les pensées évoquées : ses incertitudes sur sa capacité à (vouloir) comprendre les faits ("Were you part of the plot, a party to it? I would like to know. I think I would like to know.", (A 2)) ; l'arbitraire de toute tentative d'interprétation générale de ces faits ("I if tried I could connect everything in a vast and secret agenda. If I tried", (A 4)) ; l'arbitraire de toute tentative de mise en récit de ces faits ("Do we really need all this, these touches of local colour and so on? Yes, we do.", (A 7)) ; et l'arbitraire de la mémoire de ces faits ("he cannot have been wearing that greatcoat yet, the weather was still too warm; if I have got that detail wrong, what else am I misremembering?", (A 12)) sont mis en scène dès les premières pages du récit. C'est que Freddie est conscient du regard solipsiste qu'il pose sur le monde et les autres, un regard qui nécessite la médiation constante de dispositifs littéraires ${ }^{30}$ ou artistiques ${ }^{31}$, à commencer, bien sûr, par la peinture ${ }^{32}$. Incapable de nouer une relation authentique, ou de se connaittre, Freddie est un Narcisse en miettes, tenant désespérément de combler par un discours hypertrophié l'abîme sous le masque de l'hypocrite :

Even if I had felt a spider's web of foreboding brush against my face I would have been drawn irresistibly through it by the force of that linked series of tiny events that began the instant I was born, if not before, and that would bundle me however unceremoniously through today's confrontation, just as it will propel me on to others more or less fateful than that one until at last I arrive at the last of all and disappear forever into the suddenly shattered mirror of my self. It is what I call my life. It is what I imagine I lead, when all the time it is leading me, like an ox to the shambles. ${ }^{33}$

Freddie n'est pas dupe de l'artifice qu'il y a à relire sa vie sous l'angle de la tragédie : ni l'interprétation des événements en termes de fatalité ou de destin (fateful) ni l'acceptation de leur contingence absolue ne le satisfont, et sa lecture de l'intrigue d'Athena (the plot, comme il le dit) hésite constamment entre les deux.

Face à ce règne de l'incertitude et du faux (fausses identités, faux tableaux, mensonges et trahisons), l'art semble offrir la seule forme de réponse possible. Aussi la lettre d'amour à A. que constitue le roman (il s'ouvre par "My love" et se termine par "Write to me, she said. Write to me. I have written") est-elle entrecoupée, dans des chapitres à part qui scandent le récit, par les sept ekphraseis de Freddie où, pastichant le style du catalogue raisonné, il entreprend le commentaire des tableaux de la collection. On s'attendrait à ce qu'il se produise une double substitution : au récit intime en première personne, où la sincérité du discours est rendue impossible par la conscience aiguë du narrateur de l'artificialité de sa parole, doit se substituer le discours scientifique, en troisième personne, dans un langage normé, transparent et efficace; la certitude de l'analyse et la confiance de l'expert en ses capacités doivent remplacer le désespoir de l'amoureux trahi et du Narcisse isolé. Or, ne cessent de se produire entre ces deux régimes de discours des effets de contamination. Les tableaux, représentant des scènes mythologiques tirées des Métamorphoses, et autant de femmes sur le point d'être violées, enlevées ou perdues, semblent résumer allégoriquement les événements des chapitres qui les précèdent ${ }^{34}$. Seul personnage masculin, un Ganymède, "a curiosity among this curious collection" (A 129), subit le même sort. La notice qui lui est consacrée semble d'abord respecter les principes d'écriture du genre : objectivité du ton, érudition du commentaire, technicité des termes employés : "We detect influences 
as disparate as Tintoretto, in the dash and dramatic pace of the piece, and Parmigiano in the curious elongation of the figures" (A 129.). Mais le commentateur tombe dans le jugement axiologique et étend son soupçon (pour Freddie, all the world's a stage, et tous les hommes et toutes les femmes ne sont que des hypocrites) à l'œuvre elle-même : "We do not quite credit his [le père de Ganymède] grief. He has the air of a man who knows he is being looked at and that much is expected of him" (A 130). Surtout, abruptement, la première personne se substitue à la troisième et la description embraye à nouveau sur la lettre intime: "Those tears: he must have painted them with a brush made of a single sable hair. Remember when I showed them to you through the magnifying glass?" (A 130). Enfin, la notice se termine sur un parallèle évident entre les tourments de Freddie et ceux du peintre: "Some little time after completing this painting van Ohlbijn [...], so it is said, abandoned by his mistress, drank potion from a gilded cup and died on the eve of his forty-eight birthday. The gods have a sense of humour but no mercy" (A 131). Les effets de contamination sont par la suite de plus en plus marqués : la notice d'Acis and Galatea se conclut ainsi: "I am Acis and Polyphemus in one. This is my clumsy song, the song the cyclops sang" (A 204). L'ekphrasis érudite, le discours scientifique, sont devenus partie intégrante du lyrisme amoureux déployé dans le roman ; fausseté des œuvres et hypocrisie du correspondant se répondent.

Dans un article consacré au genre de la confession dans le roman anglophone ${ }^{35}$, Elke D'hoker souligne que la sécularisation du discours confessionnel dans la fiction contemporaine pose le problème de son efficacité : en absence de toute autorité apte à délivrer une absolution, l'aboutissement de la confession ne peut résider que dans un déplacement du repentir vers l'expression d'un désir de vérité, tandis que le pardon est remplacé par un pacte de sincérité avec le lecteur. Or ce déplacement engendre une écriture et une lecture du soupçon: comment faire la part du discours vrai et de la complaisance ? Où arrêter la confession, sans tiers qui lui fixe des limites par son ego te absolvo ? Il nous semble que la médiation du discours érudit sur la collection offre dans Athena une solution originale à l'aporie, en renversant la conception augustinienne de la sincérité de la confession: non plus dire ce qu'on croit vrai dans le but de le faire croire, mais dire ce qu'on sait ne pas l'être et jouer à y croire, en espérant peut-être y parvenir. La production d'un discours sur soi sincère de Freddie ne passe plus par l'aveu du meurtre, explicite dans le premier tome, alors qu'il est voilé dans le dernier, mais par l'expression d'une double défaite, amoureuse et esthétique, convertie en chant d'amour. L'inauthenticité foncière du narrateur ne lui laisse d'autre choix que d'adopter une forme de sincérité du masque - d'adhésion à soi par la performance d'une croyance dans l'efficacité, dans la réalité, de ce masque. C'est la qualité qu'il reconnait à son Aunt Corky: "She lied with such simplicity and sincere conviction that really it was not lying at all but a sort of continuing reinvention of the self" (A 22). Lorsqu'elle meurt, il lui réserve l'oraison suivante :

This was a world without Aunt Corky in it. What had been her was gone, dispersed like smoke. Forgive me, Auntie, but there was something invigorating in the thought; not the thought that you were no more, you understand, but that so much that was not you remained. No, I do not understand it either but I cannot think how else to put it. (A 205)

12 On note que la préciosité ("orotund quality") qui signale ses mensonges a disparu, au profit de répétitions et de phrases un peu maladroites. Si Freddie peut nouer une relation véritable avec sa tante, même post-mortem (elle est ici l'autre you du texte, le pronom renvoyant majoritairement à A.), c'est parce qu'elle incarne la possibilité d'un 
être en constante performance de soi, mais disparaissant avec son œuvre : la somme de ses masques. Elle n'existe que dans le discours qu'elle tient sur elle-même. Et Freddie ne peut dire son rapport à soi et au monde que par le truchement d'un discours sur l'art, n'exister que dans ce discours. La notice de catalogue fonctionne, pour une part, sur un modèle perecquien : produite à partir de générateurs textuels (les noms des peintres sont tous des anagrammes approximatives de John Banville; ce sont des pastiches de discours de savoirs; les commentaires, présentés comme des ekphraseis, sont en fait tirés d'un autre texte, Les métamorphoses), c'est en partie un outil de mystification. Plus Freddie commente les tableaux, moins il s'aperçoit qu'ils sont faux ${ }^{36}$. Mais la visée du pastiche dépasse "le seul frisson du faire-semblant" : il est le lieu où le narrateur peut le mieux renverser les mensonges de son discours en parole intime. On peut donc se poser la question de ce à quoi renvoie sa déclaration finale, "I have written" : la lettre à A./art est-elle le récit qu'on vient de lire, ou le catalogue de la fausse collection? Dès lors, l'effet de contamination entre notices et lettre ne relèverait plus du manque de maitrise d'un scripteur qui se laisserait surprendre par un sentimentalisme maladroit, mais d'une stratégie par laquelle le texte scientifique est miné par le chant amoureux, et le chant amoureux parvient à une fragile authenticité à travers les erreurs du texte scientifique. Car la seule garantie de sincérité de la parole de Freddie tient dans l'aveu de la totalité de son échec : échec de son regard masculin réifiant posé sur une femme qui l'égare, échec de son regard de connaisseur sur des faux, échec de sa maîtrise du discours. C'est pourquoi la Naissance d'Athéna est le seul tableau authentique : Freddie a enfin renoncé à enfermer dans les méandres de son esprit, et de sa parole, celle qui pourrait être son autre.

13 Ce paradoxe de l'entrée de catalogue comme lieu textuel où le narrateur, mélancolique, cherche une forme de certitude, d'objectivité, mais qui s'avère le lieu d'émergence de sa subjectivité troublée, dans une performance de soi à la fois hypocrite et sincère, se retrouve dans le roman de C. Portier, De toutes pièces. Le texte se présente comme l'inventaire d'un expert à qui on donne des moyens illimités pour construire une collection idéale : chaque nouvel expôt intégré à la collection y fait l'objet d'une entrée datée. Cependant, l'inventaire glisse très vite vers le journal, comme le curateur ne cesse d'en faire la remarque ("Mais je m'épanche trop. Ce carnet doit rester le lieu de consignation nécessaire des pièces reçues et de mes intentions objectives.", (DTP 29)). On retrouve tous les ingrédients contemporains du récit de collection: un collectionneur mystérieux ${ }^{37}$; un curateur aliéné, dépossédé de la collection dont il a la charge ${ }^{38}$ et porté au mensonge ; la dispersion programmée de la collection (comme dans Veuves au maquillage, le narrateur s'ingénie à envoyer des colis par surprise, jouant avec la valeur d'échange des objets et les destinataires ${ }^{39}$ ). À nouveau, la collection a vocation à fonctionner comme un microcosme capable de donner à lire le monde sous la forme de l'anatomie : "J'aimerais que ce cabinet de curiosité [...] sache proposer du monde un dessin anatomique" (DTP 102). Et, à nouveau, le lien référentiel et épistémique entre macrocosme et microcosme est rompu; le monde demeure illisible et la collection le devient avec lui. Car le dispositif même de constitution de la collection est aliénant: pouvant sans aucune limite choisir tous les objets à sa fantaisie, le curateur est rapidement confronté à l'arbitraire de sa démarche :

Je ne communique avec eux [les commanditaires] que par l'intermédiaire d'une interface web [...] : une fois validée, la pièce enregistrée disparaît de l'écran, tout redevient lisse et vide, et ne m'appartient plus. [...] Rien ne m'est laissé comme 
preuve de ce que je transmets [...], comme si plus rien n'était que fabulation de ma part [...]. Mon tableau n'a plus aucun référent. (DTP 33-34)

\section{la fois contingente ${ }^{40}$ et piégée, à l'image du miroir "escamoteur" que le curateur y} introduit :

Il ressemble à celui que l'on voit dans Le Prêteur et sa Femme de Quentin Metsys [...]. Ce sont des miroirs de vérité. [...] Mais ce miroir, petit chef-d'œuvre d'optique que j'ai trouvé là, ne rend ni détail piquant, ni hors-champ qu'un angle trop aigu cacherait à la vue. [...] Seulement, placé bien en face du miroir et au centre de la pièce, le regardeur sera pris d'une sorte de malaise. [...] Lui dans ce décor ? Il sera comme absenté, nié tout simplement. Comme si ce n'était pas lui qui venait là pour regarder, comme si c'était lui qui était placé là pour disparaître. Oh, ce sera beau comme du Méliès, faire apparaître ainsi un spectre sur la souple pellicule du réel! (DTP 23-24)

Bien sûr, le piège destiné au commanditaire finit par symboliser la position même du curateur: Narcisse sans son miroir de vérité, incapable de se rapporter au réel autrement que par une série de médiations visuelles (peinture et cinéma), fantôme dans un univers de trompe-l'œil. L'échec de la collection provoque en lui une triple crise, du savoir, de la parole intime, du rapport au monde :

Quand je fais cet inventaire, le monde parle par ma bouche. Je reconnais tout, d'avoir tout passé sous ma langue, comme autant d'étiquettes à coller, à refourguer. Mais ça ne suffit pas. La langue m'échappe. La langue des noms, vulgaires et latins, la langue où s'encaissent et s'empilent des dérivations de taxons, toute la substance des noms collée par-dessus les choses se met à fondre, tout se décolle. (DTP 117)

Comme dans Athena, le curateur-Narcisse est hanté par le rêve récurrent d'une femme mystérieuse qui lui échappe, et à qui fait pendant un second personnage féminin, peu mis en valeur ("la jupe un peu trop courte de la serveuse [...]. Ça sert un peu, ça ne boudine pas encore" (DTP 149)), mais qui lui permet de maintenir un lien, ténu, avec le réel : “Les êtres me tombent des mains. Seuls leurs vestiges m'intéressent. Le visage de la serveuse ne revient pas, voilà ce que j'ai laissé là-bas. Je n'ai rien vu, en fait. Je ne sais pas voir. Je ne fais que jauger" (DTP 150). Le remède à la mélancolie et au solipsisme du narrateur prendra alors trois formes: la falsification, la formation du regard, l'acceptation de l'altérité sous la forme de la fiction.

La pratique de la falsification est une résistance au mercantilisme des collectionneurs, voire une véritable éthique professionnelle, l'exercice d'une liberté retrouvée ${ }^{41}$ :

Je suis là pour ça, écrire des légendes. Les apposer en petite police sur des étiquettes et faire que depuis ce tout petit encart, le nom des choses grossisse, grossisse, grossisse, au point de tout envahir, au point de prendre la place des choses ellesmêmes, et du souvenir de leur origine. [...] J'amplifie, je déforme, je fais mon travail. (DTP 109)

Je suis l'artisan et la victime d'un système fondé sur la croyance, qui en même temps la mine. Je voudrais apprivoiser ce processus. Le retourner une dernière fois, faire que l'arroseur soit arrosé. [...] Je pense avoir retrouvé ma marge de liberté dans cette demande folle. (DTP 134)

Mais, dans De toutes pièces, l'expérience émancipatrice de l'altérité se fait surtout par le truchement d'un chat, qui, déambulant dans l'entrepôt où sont stockées les pièces de la collection, force le curateur à accepter l'irruption de l'imprévu et le plaisir de la surprise. Un jour, il surprend l'un des gardiens à faire semblant de tirer sur le chat pour le tuer, puis le chat disparaît. Dès lors, l'espace de la collection, organisé par le curateur pour résumer un monde qu'il contrôle et momifie par son entreprise constante de 
nomination, d'étiquetage et de classification, devient le lieu où le jeu et le vrai, la fiction et le réel, se confondent: "Il se peut aussi que le chat soit mort. Que la fiction soit performative et qu'une volonté de nuire suffise à faire mourir un chat [...]. Mettons que le chat soit mort. Il entrerait alors dans mon domaine : celui des choses inanimées. Je saurais alors mieux le traiter, car c'en serait fini du régime de la surprise dans lequel cette bête évolue" (DTP 93). Quand le narrateur décide de sortir de son isolement, il prolonge le geste de falsification, qui minait la collection de l'intérieur, par un acte plus radical encore : la dispersion de la collection ${ }^{42}$, c'est-à-dire le refus de l'attitude au monde qu'elle symbolise, un regard masculin prédateur confondant les êtres et les choses, une entreprise de prélèvement et de possession, l'autorité d'un savoir qui réduit le monde à un spectacle narcissique. Son changement de regard est symbolisé par son rapport à l'animal :

Le chat. [...] Je ne lui donnerai pas de nom. Je ne lui parlerai pas. Je ne chercherai pas à l'accaparer, à le séquestrer dans mon monde qui transforme les êtres et les choses en objets à sa langue et à sa main. (DTP 133)

Et le roman de se conclure : “Je vais partir, laisser les objets non liquidés. [...] J'emporte le chat. Je laisse la porte ouverte" (DTP 174).

\section{Fictions à crédit}

Vers la fin de sa lettre, Freddie désabusé s'interroge : “Since I am no longer speaking to anyone except myself [...], I do not know why I should go on fussing over niceties of narrative structure, but I do" (A 211-212). Les deux narrateurs d'Athena et De toutes pièces se servent du truchement de discours normés (catalogue, inventaire) pour se construire des identités fragiles. Les lieux textuels du savoir deviennent ainsi, sous leur plume, des espaces de mensonge et de fiction. Ils n'entendent pas convaincre leurs narrataires mais proposer une pratique assumée du faux comme seule forme possible d'authenticité. Dès lors, ils ne leur demandent pas tant de les croire que d'accorder leur attention aux fictions qu'ils élaborent. Si Freddie prête encore intérêt aux fioritures de la narration, c'est parce que le seul crédit dont il peut bénéficier est celui qu'il s'octroie comme narrateur, comme storyteller. De même le curateur de C. Portier commente-t-il ironiquement: "on préfère toujours être un salaud qui trompe qu'un salaud qui assassine" (DTP 63), ce qui pourrait valoir comme résumé grinçant d'Athena : Freddie s'y construit par le mensonge sa rédemption d'ancien meurtrier. Tous deux pratiquent une sorte d'éthique ambiguë du mensonge: "Mensonge est parole cloisonnée. J'ai, moi aussi, mes petites boîtes à mensonges" (DTP 43). La fiction, dans ces textes, ce serait le "décloisonnement" du mensonge, à travers une remise en circulation des images, l'ouverture de boîtes de Pandore textuelles. Ainsi, significativement, dans la trilogie de Banville, des personnages issus d'autres ouvrages reviennent, brouillant la clôture du texte et de l'univers fictionnel qu'il met en place ${ }^{43}$. De même, on observe une migration des images dans le travail de C. Portier : le "cil de Marylin Monroe [...] prélevé à même son cadavre barbiture" (DTP 41), ou la photographie de la petite fille morte qui fascine le narrateur, sont pris à des œuvres antérieures ${ }^{44}$. Les prélèvements, textuels et iconiques, tirés d'œuvres précédentes font des suivantes non plus une collection fermée mais des livres ouverts : la migration des signes et des images est une entreprise de resémantisation et de partage de ces contenus ${ }^{45}$. Le mensonge n'en est pas moins présent, mais il est décloisonné. Aussi le curateur peut-il affirmer à la fois: "Un 
souvenir, par exemple, est une chose fiable" (DTP 31), et : “J'oublie beaucoup de choses, voilà pourquoi je note. J'oublie ce qui m'affecte" (DTP 26).

Les narrateurs des récits de collection produisent par l'écriture une identité performée qui, comme la fiction du chat, peut bien devenir performative. Le mensonge pratiqué avec assiduité et la copie, la reprise, peuvent alors devenir, peut-être, de paradoxaux régimes d'authenticité. Ce n'est pas un gage parfait: les fins demeurent ambiguës, comme la capacité des personnages à se défaire de leur "complexe d'Actéon" ${ }^{46}$. A., la serveuse ou le chat ne sont pas les représentations de l'altérité les plus convaincantes, et l'acceptation de l'échec, sous la forme de l'écriture de la lettre d'amour ou de la dispersion de la collection, n'est pas une ferme promesse d'émancipation. Mais le mélange de mélancolie, d'ironie et d'humour ${ }^{47}$ de ces discours, la tentative désespérée de production d'un récit de soi malgré l'éclatement du moi, le détournement ironique de la confession en performance de fausses identités en série, plaident tous la même cause : accorder crédit, sinon créance, au mensonge de la fiction, au bal des masques.

\section{NOTES DE FIN}

1. Pour reprendre le terme adopté par C. Portier dans De toutes pièces, Meudon, Quidam, 2018 (dorénavant DTP).

2. Sur le traitement de la figure de l'historien de l'art dans le roman contemporain, voir Nella Arambasin, Littérature contemporaine et "histoires" de l'art : récits d'une réévaluation, Genève, Droz, 2007.

3. "ce qu'est fondamentalement l'imposture: posture usurpée, une attitude plus souvent subie que choisie, un carcan, une camisole de force", précise Maxime Decout dans Pouvoirs de l'imposture (Paris, Éditions de Minuit, <Paradoxe>, 2018, p. 8-9), qui analyse justement les liens entre la question du savoir et de l'imposture.

4. DTP 43.

5. Pierre Senges, Veuves au maquillage, Paris, Verticales, 2000, p. 249 (dorénavant VM).

6. Sur la mauvaise foi en littérature, voir Maxime Decout, En toute mauvaise foi, Paris, Éditions de Minuit, 2015, <Paradoxe>.

7. Georges Perec, Un cabinet d'amateur, Paris, Seuil, 2001 [1979], <Points>, p. 9 (dorénavant CA).

8. Le catalogue de la vente Raffke mêle éléments réels et fictifs : Charles M. Murphy s'attaquant au record du mile le 30 juin 1899 est une toile de Bernie Bickford, censé avoir travaillé dans l'atelier de Bonnat, rencontré le "gangster notoire" Angelo Merisi, et peint des tableaux conservés au Police Academy Museum de Brooklyn (CA 31). Si le peintre est fictif, Murphy existe, Bonnat aussi, il y a bien un Police Academy Museum à New York, et Michelangelo Merisi n'est autre que le Caravage, connu pour ses déboires avec la justice de son temps : le texte trouble les frontières entre fiction et réalité, désamorce le fonctionnement correct de la référence.

9. Sur l'usage de passages de La vie mode d'emploi comme "générateurs textuels" des tableaux de la collection, voir Bernard Magné, “Peinturecriture", Perecollages 1981-1988, Toulouse, Presses universitaires du Mirail, 1989.

10. Sur le fonctionnement de "l'impli-citation", voir ibid., p. 74 notamment. 
11. Voir la phrase finale ("Des vérifications entreprises avec diligence ne tardèrent pas à démontrer qu'en effet la plupart des tableaux de la collection Raffke étaient faux, comme sont faux la plupart des détails de ce récit fictif, conçu pour le seul plaisir, et le seul frisson, du fairesemblant", (CA 85)).

12. Perec a exposé les liens entre la pratique du trompe-l'œil et sa propre écriture dans une préface à l'édition des photographies de $\mathrm{C}$. White: “Ce que, en fin de compte, le peintre de trompe-l'œil nous dit, ce qui déclenche en nous ce petit vertige n'est rien d'autre que : 'Ceci n'est pas un mur.' Or, bien sûr, si la pipe de Magritte n'est pas une pipe, puisqu'elle n'est, tout simplement, tout bonnement, qu'un peu de peinture étalée sur une toile, le mur peint en trompel'œil est, lui, bel et bien un mur. Il n'est même que cela : mur nu, sans relief, sans ouvertures, sans corniches, sans rebords saillants, pur obstacle que le simulacre de la peinture essaye de faire passer pour quelque chose qu'il n'est pas." Georges Perec et Cuchi White, L'œil ébloui, Paris, Chêne, 1981, texte non paginé.

13. Le Cabinet d'amateur serait ainsi "l'expression ultime de la mélancolie de l'artiste" - si toutefois on prête foi aux propos de Nowak (CA 28).

14. VM 164.

15. Voir Audrey Camus, "Anatomie de la fiction : Veuves au maquillage de Pierre Senges", Littérature, vol. 151, n 3, 2008, p. 22 : "Pierre Senges, nouveau Frankenstein, donne ici le jour à une étrange créature, dans une figuration spectaculaire de l'entrée en littérature de l'écrivain sacrifiant sa dépouille sur l'autel de la fiction."

16. C'est la thèse défendue par A. Camus (ibid.).

17. VM 83.

18. Pierre Senges se revendique notamment de Giorgio Manganelli (voir par exemple La littérature comme mensonge, trad. Philippe Di Meo, Paris, Gallimard, 1991, p. 63 : "Rien n'est plus mortifiant que de voir des romanciers qui [...] s'abandonnent aux rêves morbides d'une transcription documentaire, éducative ou pathétique de la réalité. [...] Bien qu'ils soient forcés de mentir, comme l'exigent les lois punitives des lettres, ils le font avec angoisse et mauvaise conscience : on les voit souffrir sous la contrainte de la fraude, et c'est en vain qu'ils dissimulent un authentique nœud de mensonges sous le voile d'une fictive ressemblance.")

19. Le terme est à prendre dans son sens étymologique de dissimulation de son identité par imitation de celle d'un autre. Il s'agit de jouer à être quelqu'un d'autre, pour exhiber et cacher la fragilité, voire l'inexistence, de l'identité de celui qui se disperse en ses masques. Voir aussi Maxime Decout, En toute mauvaise foi, op. cit.

20. Michèle Sinapi, “D'un mensonge l'autre : mensonge privé, mensonge politique. Le tournant kantien", dans Françoise Reumaux (dir.), Passeports pour le vrai/le faux, Paris, Kimé, 2005, p. 38. 21. Ibid.

22. Sur la collection comme entreprise de sublimation du désir (notamment érotique) du collectionneur dans Le cousin Pons, voir Pierre-Marc de Biasi, Art. cit.

23. John Banville, The book of Evidence, Londres, Minerva, 1990 [1989], p. 215.

24. John Banville, Ghosts, Londres, Picador, 2011 [1993], p. 239.

25. Sur l'incertitude de la réalité de l'existence de A., et sur les conséquences de cette ambiguïé sur l'interprétation de l'intrigue, voir Brendan McNamee, "Interpretation, suspension, response : a reading of John Banville's Athena", Orbis Litterarum, vol. 61, $\mathrm{n}^{\circ}$ 3, 2006, p. 183-201.

26. John Banville, Athena, Londres, Picador, 2010 [1995] (dorénavant A), p. 233: "There is the she who is gone, [...] lost to me forever, and then there is this other, who steps out of my head and goes hurrying off along the sunlit pavements to do I don't know what. To live. If I can call it living; and I shall."

27. Le roman programme l'impossibilité de trancher, et la question fait débat parmi les spécialistes. Voir, notamment, deux études, sous l'angle du féminisme, qui nuancent fortement la possibilité de la rédemption de Freddie: Anja Müller, "You have been framed: the function of 
ekphrasis for the representation of women in John Banville's trilogy (The Book of Evidence, Ghosts, Athena)", Studies in the Novel, vol.36, $\mathrm{n}^{\circ} 2$, 2004, p. 185-205; Patricia Coughlan, "Banville, the Feminine, and the Scenes of Eros", Irish University Review, vol. 36, $\mathrm{n}^{\circ}$ 1, 2006, p. 81-101.

28. Par exemple, cette scène où le personnage de Francie se saisit d'un marteau, ce qui rappelle le meurtre du premier tome: "Francie ambled forward and picked up a miniature hammer from the workbench and turned toward me and - Enough of this. I do not like it down here! I do not like it at all. A wave of my wand and pop! here we are magically at street level again" (A 55). L'interruption volontaire du narrateur, comiquement soulignée par l'onomatopée, exhibe autant qu'elle le dissimule le souvenir du meurtre ; le narrateur joue à se trahir lui-même.

29. "Perhaps it was merely out of curiosity then that I- Ah, what a giveaway it is, I've noticed it before, the orotund quality that sets in when I begin consciously to dissemble [...]. Whenever I employ locutions such as that you will know I am inventing. But then, when do I not use such locutions?" (A 24).

30. "You see? - a lost love, a locked room, a now a will: we are in familiar territory after all", (A 4).

31. "The occasion of our first meeting retains in my memory a sort of lurid, phosphorescent glow; I have the impression of a greenish light and dispersing stage smoke and the sudden swirl and crack of a cloak and a big voice booming out: Tarraa!", (A 6). Sur l'importance de la référence théâtrale pour Freddie, voir Wit Pietrzak, "A Stage-Hand, Perhaps: Life as a Stage Play in John Banville's The Book of Evidence", Critique: Studies in Contemporary Fiction, vol. 61, $\mathrm{n}^{\circ} 5,2020$, p. 568-576.

32. Le lien entre l'obsession artistique, la complexité du point de vue éthique de Freddie, et les difficultés de son rapport au réel, constitue sans doute l'enjeu principal auquel s'intéressent les spécialistes de Banville. Outre les monographies pionnières de Rüdiger Imhof, John Banville: A Critical Introduction, Dublin, Wolfhound Press, 1997 et de Joseph McMinn, The Supreme Fictions of John Banville, Manchester, Manchester University Press, 1999, les travaux suivants envisagent la question: Tony E. Jackson, "Science, Art, and the Shipwreck of Knowledge: The Novels of John Banville”, Contemporary Literature, vol. 38, n 3, 1997, p. 510-533; Elke D'hoker, Visions of Alterity: Representation in the Works of John Banville, Amsterdam, Rodopi, 2004; John Kenny, "Well Said Well Seen: The Pictorial Paradigm in John Banville's Fiction”, Irish University Review, vol. 36, $\mathrm{n}^{\circ} 1,2006$, p. 52-67; Mark O'Connell, “On Not Being Found: A Winnicottian Reading of John Banville's Ghosts and Athena", Studies in the Novel, vol. 43, n 3, 2011, p. 328-342.

33. A 10 ; nous soulignons.

34. Pour une analyse précise des tableaux et de leur inscription dans l'intrigue, voir Brendan McNamee, Art. cit.

35. Elke D'hoker, “Confession and Atonement in Contemporary Fiction: J. M. Coetzee, John Banville, and Ian McEwan", Critique: Studies in Contemporary Fiction, vol. 48, n 1, 2006, p. 31-43.

36. Pourtant, à en croire Francie, leur inauthenticité est évidente: "We dried them under the lamps, he said, they were still sticky when he showed them to you and you never noticed" ( $A$ 217).

37. Non pas un criminel, comme dans Athena, ou un mort au tombeau, comme dans Un cabinet d'amateur, mais de riches anonymes dont on ne saura rien, sinon qu'ils veulent l'équivalent contemporain d'un prestigieux cabinet de curiosités, sans doute avant tout pour pouvoir spéculer.

38. "Je suis en quelque sorte un occupant sans titre, et donc sans pouvoir" (DTP 68). Il ne peut donc plus prendre à son compte les six étapes de constitution et de jouissance de la collection désignées par P.-M. de Biasi, ce qui fausse son rapport à la collection et frappe sa démarche d'inauthenticité.

39. Par exemple, à une petite fille qui devait recevoir une licorne en peluche, il offre une "licorne véritable" (DTP 159), faite d'une dent de narval ancienne fichée sur un âne taxidermisé. "C'est si 
plaisant que la mirifique ivoire, qui se vendait jusqu'à onze fois le prix de l'or, se retrouve enchâssée entre les deux oreilles d'un âne! Toute spéculation ramenée à sa bêtise, à sa possible dévaluation. Tout cela ne vaut rien et c'est ce qui en fait son prix" (DTP 161).

40. Le caractère hétéroclite et arbitraire de la collection est symbolisé par le tout premier objet de l'inventaire, dans la phrase inaugurale : "Premièrement un ornithorynque" (DTP 13).

41. Ainsi, le narrateur peut-il déclarer "Je ne supporte pas qu'on remette en cause mon professionnalisme. C'est ma seule sincérité" (DTP 85), et, à la page suivante "Je me suis donc prêté, non sans satisfaction, dans un certain esprit de revanche, à une contrefaçon d'amateur" (DTP 86).

42. Il y a un renversement par rapport au XIX ${ }^{\mathrm{e}}$ siècle à cet égard : pour Pons ou Nemo, la fin de la collection a quelque chose de tragique. Dans les récits contemporains, en revanche, la destruction de la collection a valeur émancipatrice, même si la mélancolie n'en est pas absente.

43. Dans Athena, le nom de l'inspecteur est une version déformée de celui du premier tome, et le peintre de Ghosts, Vaublin, fait une réapparition. L. Louvel a analysé ce jeu en détail dans Ghosts : "Fantômes dans le texte, ils sont aussi fantômes d'autres textes puisque les nombreux échos entre les romans précédents de Banville font de ce livre un lieu bien sonore : [...] Mefisto fournit l'original de Felix, de Licht /Leicht, du Professeur qui apparaît sous divers noms commençant par $\mathrm{K}$ : Kosok, Kasperl, Kreuznaer [...]." Liliane Louvel, “John Banville, Ghosts : l'étoffe des rêves", Études irlandaises, vol. 22, nº 1, 1997, p. 45.

44. DTP 25-26. Le cil vient d'Inventaire pour déshérence, une performance réalisée dans le cadre du festival organisé par le Général Instin, à Belleville, du 4 au 7 juin 2015. Le texte est en ligne sur le blog de C. Portier: https://petiteracine.net/wordpress/2015/06/inventaire-pour-desherence/. La photographie de la petite fille et le texte qui l'accompagne, en partie repris verbatim dans DTP, sont tirés de "Dans le viseur", l'une des séries du blog: https://petiteracine.net/wordpress/ 2015/03/le-temps-1-emporte/ (sites consultés le 6 novembre 2020).

45. Il n'est donc pas innocent que, dans DTP, les images qui circulent soient celles de corps féminins, qui échappent ainsi en partie à la prédation du curateur. Dans un entretien à paraître dans le prochain numéro de Romanesques ("Numérique et romanesque", n 13, 2021), C. Portier s'explique sur ces retours: "Ils existent surtout parce que certaines figures sont importantes pour moi à un moment, et que je les emporte avec moi d'un projet à l'autre quand je sens que je n'en ai pas fini avec elles et que je n'ai pas encore envie de les ranger".

46. Sur le complexe d'Actéon (qui relie en un même réseau le regard, le désir de savoir, le désir de vérité et le corps féminin traité comme objet), et son usage dans les fictions de Banville, voir Patricia Coughlan, Art. cit.

47. Parfois noir : voir l'entrée 11 septembre de l'inventaire, sous-titrée "jour de spectacle" (DTP 124).

\section{RÉSUMÉS}

Comment un discours scientifique aussi normé que l'inventaire de collection peut-il devenir le journal intime déguisé de menteurs professionnels? Au XIX siècle, le roman de collection, récit du martyre esthétique de collectionneurs marginaux ou galerie de portraits de tout ce que Drouot compte d'experts véreux, de faussaires sans scrupules ou de philistins dupés, met en scène des collections parfois truffées de faux, sans que la forme-collection elle-même soit considérée comme inauthentique : elle reste une forme pertinente d'organisation des objets et des connaissances. Quand la fiction contemporaine s'empare du genre, cependant, c'est pour donner une tout autre vision de la forme-collection. Celle-ci n'est plus le résultat du désir fou mais sincère d'un esthète, mais plutôt de la spéculation financière de collectionneurs anonymes peu soucieux d'authenticité. Détachée d'un projet personnel qui lui donne sens, associant objets 
authentiques et falsifiés, la collection n'est plus qu'un trompe-l'œil qui imite des discours de savoir ou d'authentification pour mieux en révéler la fragilité. Dès lors, son curateur, qui ne la possède plus, mais en est seulement le gardien et le garant, ne peut plus être qu'un menteur. Face au délitement de la collection devenue système de signes qui tourne à vide, son seul recours est de "se montrer radical dans la maitrise de l'imposture", de performer dans son inventaire une hypocrisie réglée, pour recréer, par-delà l'angoisse de la collection comme pur simulacre, un nouveau rapport aux objets - donc à soi. L'écriture des inventaires inauthentiques de la collection devient ainsi paradoxale écriture de soi, reconquête par le mensonge du rapport aux objets et aux autres, au monde dont ils sont issus, au langage qui les identifie. Se joue ainsi l'un des horizons éthiques du roman contemporain, dans la tentative de construire de fragiles relations de confiance entre narrateurs et narrataires - autant de pactes de lecture en abyme, fuyants, mais proposant une forme de crédit fictionnel.

INDEX

Mots-clés : collection, authenticité, éthique de la fiction, crédit, écriture de soi

\section{AUTEUR}

\section{LOÏSE LELEVÉ}

Université Rennes 2 (Cellam) 